\title{
Primeros pasos hacia la internacionalización en la Universidad Michoacana de San Nicolás de Hidalgo
}

\author{
First steps towards internationalization in the Universidad Michoacana de San Nicolás de Hidalgo \\ Rosa Vega Cano
}

\begin{abstract}
RESUMEN
La internacionalización se ha convertido en una de las principales tendencias de la educación superior y un imperativo para que estas instituciones puedan responder a los cambios y adaptarse a su entorno, razón por la cual este trabajo se planteó como objetivo estudiar de qué manera se está abordando la dimensión internacional en la Universidad Michoacana de San Nicolás de Hidalgo. Para ello se siguió un enfoque metodológico cualitativo; se revisaron documentos y bases de datos institucionales y se realizaron entrevistas semiestructuradas al personal de la Dirección de Vinculación y Desarrollo, área administrativa en la que se concentra información sobre el tema. La conclusión general que deriva es que esta universidad no ha integrado la dimensión internacional en sus funciones sustantivas, cuando mucho ha implementado algunos programas de internacionalización de manera marginal, aislada y sin un objetivo claro, y que para sentar las bases e impulsar el proceso de internacionalización es necesario llevar a cabo una reconfiguración académica, organizativa, administrativa y política, lo que constituye, al mismo tiempo, el principal reto al que tenga que enfrentarse esta institución.
\end{abstract}

Palabras clave: educación comparada, enseñanza superior, gestión educacional, política educacional.

\begin{abstract}
Internationalization has become one of the main trends in higher education and an imperative for these institutions to be able to respond to changes and adapt to their environment, which is why this work aimed to study how the international dimension is being addressed at the Universidad Michoacana de San Nicolás de Hidalgo. To do this, a qualitative methodological approach was followed; institutional documents and databases were reviewed, and semi-structured interviews were conducted with the staff of the Department of Linking and Development, an administrative area in which information on the subject is concentrated. The general conclusion resulted is that this University has not integrated the international dimension into its substantive functions, at most it has implemented some internationalization programs in a marginal way, isolated and without a clear objective, and that to lay the foundations and promote the internationalization process it is necessary to carry out an academic, organizational, administrative and political reconfiguration, which constitutes, at the same time, the main challenge that this institution has to face.
\end{abstract}

Keywords: comparative education, higher education, educational management, educational policy. 


\section{INTRODUCCIÓN}

La sociedad global actual, caracterizada por la interdependencia, la multiculturalidad y la pluralización, está enfrentándose a un proceso de desarrollo contradictorio en el que la globalización asume un papel protagónico, promoviendo, por un lado, la homogeneización e integración multidimensional, apoyándose en las nuevas tecnologías de la información y comunicación y, por otro, desencadenando desigualdades y divergencias entre los países.

Esta dualidad del entorno actual ha propiciado la necesidad de generar nuevos esquemas de comprensión de la realidad social que permitan actuar de manera más eficaz y eficiente ante los retos que impone la globalización, rebasando las visiones parciales y extremas que pugnan solo por lo local, o bien solo por lo global, y reconociendo que estos son fenómenos que ocurren de manera simultánea y que no son mutuamente excluyentes.

Madera (2018, p. 89) indicó: "El siglo XXI requiere cambios e innovaciones en los modelos y sistemas educativos, en su articulación con la dinámica local, regional y global, en las instituciones educativas, sus procesos sustantivos y en el perfil de los actores académicos", y la internacionalización de la educación superior, entendida como el proceso mediante el cual las universidades integran los componentes global e intercultural en sus funciones sustantivas (Knight, 2012), constituye una alternativa de transformación tanto a nivel institucional (ya que requiere del involucramiento de todos los niveles y actores que forman parte de la comunidad universitaria) como para la sociedad en general, pues dicho proceso se sostiene en la resignificación y revaloración de la historia, la cultura y las tradiciones de los países, así como en el respeto de sus diversidades.

De esta forma, la integración transversal de la dimensión internacional en las universidades hace posible que el conocimiento que se genera, transmite y transfiere desde ellas sea socialmente pertinente, que contribuya a contrarrestar los efectos negativos de la globalización y a la construcción de escenarios propicios para el desarrollo sostenible.

En ese sentido, la internacionalización se ha convertido en una de las principales tendencias de la educación superior (Tünnermann, 2018) y en un imperativo para que estas instituciones puedan responder a los cambios y adaptarse a su entorno.

Rosa Vega Cano. Profesora-investigadora de la Facultad de Economía "Vasco de Quiroga" de la Universidad Michoacana de San Nicolás de Hidalgo, México. Es doctora en Gestión de la Educación Superior por el Centro Universitario de Ciencias Económico Administrativas de la Universidad de Guadalajara. Cuenta con Perfil Deseable ProdeP. Entre sus principales temas de interés se encuentran: vínculo entre la educación superior y el desarrollo sostenible, perspectiva de género en las instituciones de educación superior, responsabilidad social universitaria e internacionalización de la educación superior. Correo electrónico: rosa.vega@umich.mx. ID: https://orcid.org/0000-0002-5668-1429. 
Por tal motivo, este trabajo se planteó como objetivo estudiar de qué manera se está abordando la dimensión internacional en la Universidad Michoacana de San Nicolás de Hidalgo (en adelante Universidad Michoacana), para lo cual se revisaron documentos y bases de datos institucionales y se realizaron entrevistas semiestructuradas al personal de la Dirección de Vinculación y Desarrollo (en adelante DVD), que es el área administrativa en la que se concentra información sobre movilidad y convenios internacionales.

Si bien la Universidad Michoacana no cuenta con una política institucional de internacionalización y tampoco con una estructura formal para operar estrategias sistemáticas y orientadas hacia el cumplimiento de metas y objetivos específicos, es claro que se trata de un tema que debe ser incluido en la agenda institucional en el corto plazo. El viraje hacia la internacionalización constituye un elemento fundamental para fortalecer la calidad y pertinencia de la educación y de la investigación, procesos sustantivos del quehacer universitario (De Wit, Hunter, Howard y Egron-Polak, 2015).

El documento se compone de tres apartados, el primero describe los elementos teórico-conceptuales que se han tomado como referencia para definir los elementos que se proponen como punto de partida para impulsar el proceso de internacionalización en la Universidad Michoacana, el segundo constituye la propuesta que orientaría dicho proceso, y finalmente se ofrecen algunas reflexiones a manera de conclusión.

\section{ELEMENTOS TEÓRICO-CONCEPTUALES DE REFERENCIA PARA INICIAR UN PROCESO DE INTERNACIONALIZACIÓN}

Gacel-Ávila (2003) propuso sustentar el concepto de internacionalización de las instituciones de educación superior en dos perspectivas teóricas: a) la Teoría general de los sistemas y de las organizaciones y b) la Pedagogía crítica. La primera es fundamental para comprender cómo estas instituciones conforman un complejo entramado de actores y relaciones que interactúan tanto al interior de ellas mismas como hacia el exterior $y$, al formar parte de ese sistema, se ven afectadas por las decisiones y acciones que otros actores llevan a cabo (se trata pues de un sistema abierto), por lo que requieren de la implementación de estrategias que les permitan adaptarse a esas cambiantes características de su entorno. La Pedagogía crítica, por otra parte, aporta elementos para integrar al currículum de la educación superior componentes que permitan formar ciudadanos críticos y con conciencia global que, en el ejercicio de su profesión, se desenvuelvan como agentes proactivos de la transformación hacia un mundo más equitativo y socialmente justo; lleva a cuestionar el qué, por qué y para qué en el proceso de enseñanza-aprendizaje.

Así, la internacionalización de la educación superior puede ser entendida como un proceso permanente, contrahegemónico y comprehensivo (ver figura 1), "que 
ocurre en un contexto internacional de conocimiento, donde las universidades son vistas como un subsistema, una parte de un mundo más amplio e inclusivo [ $[. . \mathrm{y}$ que] implica un programa de acción [...] integrado en todos los aspectos y niveles educativos" (Gacel-Ávila, 2003, p. 53).

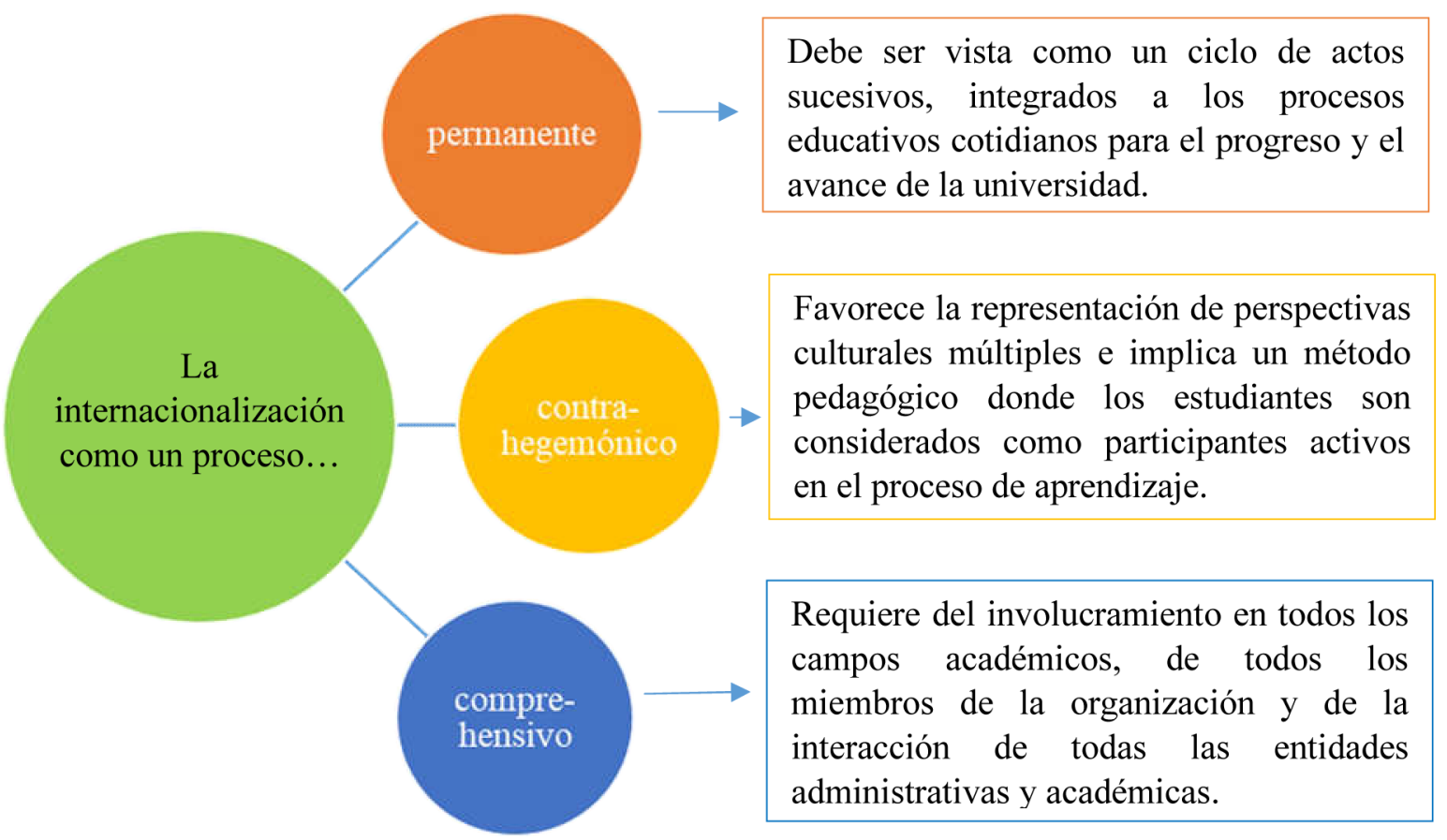

Figura 1. Elementos implícitos en el concepto de internacionalización.

Fuente: Elaboración propia con base en Gacel-Ávila (2003, pp. 53-54).

Si bien es cierto que es prácticamente imposible que las instituciones de educación superior logren adaptarse total y fácilmente a los cambios que les son impuestos por el contexto externo, la planeación estratégica constituye una herramienta que les permite estar preparadas y responder de una manera más adecuada en el ambiente de incertidumbre en el que se desenvuelven. En este sentido, De Wit (2001, citado en Gacel-Ávila, 2003, p. 331), propuso un modelo en el que enfatiza el efecto integrador y el carácter estratégico de la internacionalización en la política de desarrollo institucional de la universidad, adecuando propuestas que, desde la década de 1990, han realizado otros autores, entre los que destacan Knight, Van der Wende y Rudzki (ver figura 2).

En este modelo se sustenta el análisis que aquí se realiza en torno a la internacionalización de la Universidad Michoacana de San Nicolás de Hidalgo. 


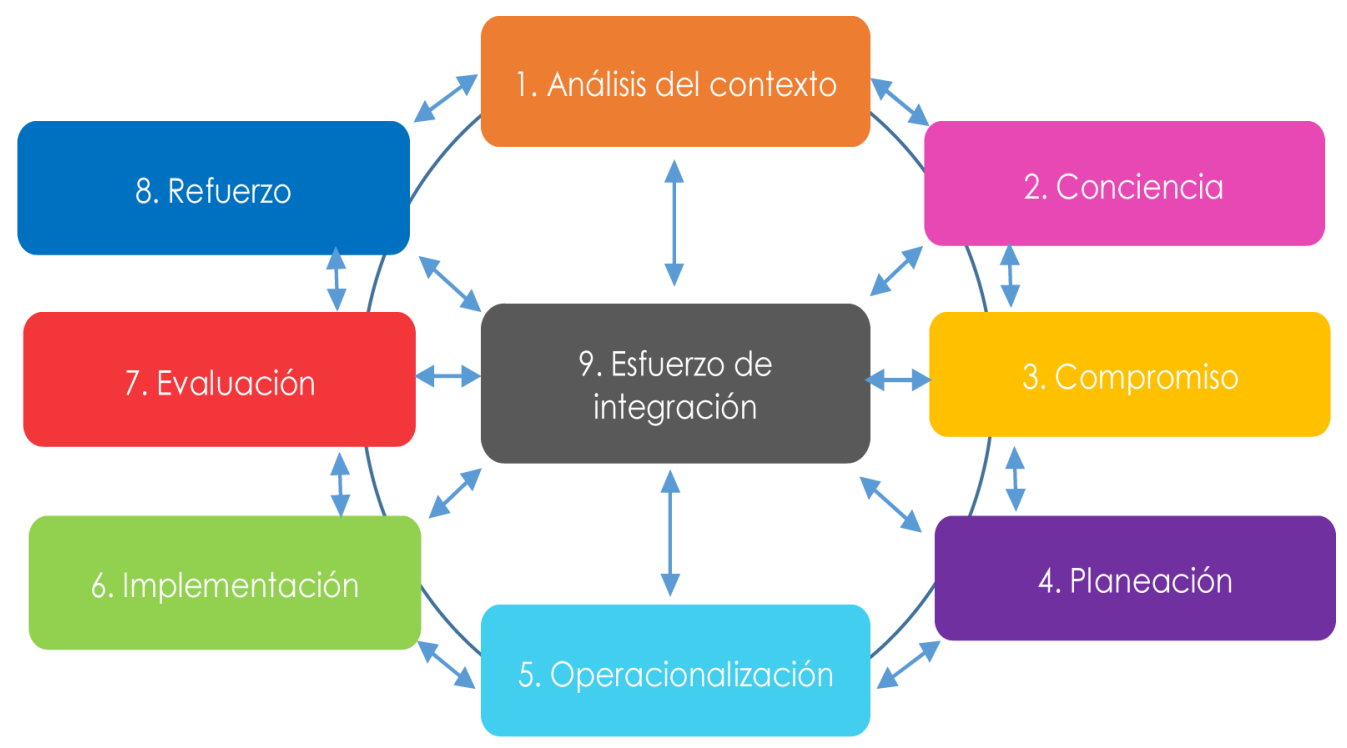

1. Análisis del contexto interno y externo en documentos de políticas.

2. Conciencia de la necesidad, objetivos y beneficios de la internacionalización para los estudiantes, académicos y administrativos.

3. Compromiso de autoridades, consejos universitarios, profesores, administrativos y estudiantes.

4. Planeación o estrategia institucional que especifique metas y objetivos en función de los intereses y las características de la propia institución.

5. Operacionalización: actividades académicas y de servicio, factores organizacionales y lineamientos.

6. Implementación de programas y estrategias internacionales.

7. Evaluación de la calidad y el impacto de las iniciativas y el progreso de las estrategias.

8. Refuerzo: desarrollo de incentivos, reconocimiento para el personal académico y participación de los estudiantes.

9. Impacto en la docencia, investigación y servicios.

Figura 2. Elementos del modelo de internacionalización propuesto por Hans De Wit (2001).

Fuente: Adaptación propia con base en Gacel-Ávila (2003, p. 332).

\section{ELEMENTOS DE PARTIDA PARA INICIAR}

\section{EL PROCESO DE INTERNACIONALIZACIÓN EN LA}

\section{Universidad Michoacana de San Nicolás de Hidalgo}

\section{Análisis del contexto}

En un entorno tan interconectado y susceptible a cambios como en el que vivimos actualmente, es imprescindible que las universidades estén atentas a lo que ocurre tanto en su interior como en el exterior para identificar qué factores podrían afectarles y de qué forma podrían enfrentarlos. De no conocer sus fortalezas y debilidades, las universidades difícilmente pueden adaptar sus procesos para resistir los retos 
y/o aprovechar las oportunidades que se les presenten (Gacel-Ávila, 2003). En este sentido, resulta de suma importancia identificar cuáles son los elementos que actúan como impulsores y cuáles como limitantes de la internacionalización, tanto al interior de la Universidad Michoacana como en México y más allá de las fronteras nacionales.

\section{El contexto externo.}

Las tendencias actuales de la educación superior han sido fuertemente influenciadas, como se ha dicho, por la globalización. De igual forma, las asimetrías derivadas de la visión economicista y neoliberal desde la cual se ha impulsado son evidentes entre los países y al interior de ellos. La "diversidad de situaciones [...] en sus esferas gubernamentales, sociales, culturales y económicas [...] impactan en el desarrollo de las políticas educativas que generalmente se aplican con distintos ritmos y prioridades" (Navarrete, 2021, p. 97).

Las múltiples desigualdades que caracterizan a la realidad social vigente, por tanto, vuelven necesario y urgente el surgimiento de planteamientos alternativos que promuevan la visión de un mundo más justo y sostenible. En este sentido, la universidad, como encargada de la generación, transmisión y transferencia del conocimiento, tiene una gran responsabilidad en términos de hacer que este sea lo suficientemente pertinente, para lograr contrarrestar los efectos negativos de la globalización y hacer que las relaciones internacionales, regionales y locales sean más equilibradas y produzcan mejores resultados para todos. Al respecto, Ordorika y Soley (2018, p. 64) señalaron que "la profundización de la valoración social de la institución universitaria constituye un elemento central para superar el reduccionismo productivista y de mercado, como fin último de la educación superior".

Uno de los procesos a través de los cuales la universidad puede incidir en la transformación social es la internacionalización, ya que se le reconoce como

...una herramienta clave para transformar la educación superior, fortalecer sus comunidades y promover la calidad y pertinencia de la docencia, la investigación y la extensión. [Puesto que] favorece la formación de ciudadanos y profesionales, respetuosos de la diversidad cultural, comprometidos con el entendimiento intercultural, la cultura de paz y con capacidad para convivir y trabajar en una comunidad local y mundial [CRES, 2018, pp. 7-8].

Gracias al reconocimiento de la importancia que tiene la internacionalización de la educación superior, gobiernos de distintos países y regiones del mundo (principalmente Europa, Asia y Norteamérica) suman esfuerzos con sus universidades para impulsarla, mediante políticas nacionales e institucionales acompañadas de financiamiento. Sin embargo, otras regiones como América Latina y el Caribe se caracterizan por tener una visión más limitada de los beneficios de la internacionalización y, por tanto, muestran un menor compromiso con su financiamiento e institucionalización; al respecto, Gacel-Ávila y Rodríguez-Rodríguez (2018a) señalaron: 
...en la región, los programas de gobierno se caracterizan por un insuficiente financiamiento, además de una falta de continuidad y coordinación entre las diferentes instancias, agencias y actores [p. 71].

En México, recientemente la Asociación Nacional de Universidades e Instituciones de Educación Superior (ANUIES) señaló que el impulso de la internacionalización es uno de los retos de mayor relevancia para la mejora continua de la calidad de la educación superior, y estableció entre sus objetivos específicos el fortalecimiento de la internacionalización de las instituciones de educación superior (IES), especificando siete líneas de acción para lograrlo (ANUIES, 2018, pp. 123-124):

- Establecer un grupo de trabajo para la elaboración del Programa Nacional de Internacionalización de la Educación Superior, integrado por académicos expertos en la materia, funcionarios de gobierno y representantes del sector empresarial.

- Incorporar en las agendas de la SEP (Secretaría de Educación Pública) y de la Conago (Conferencia Nacional de Gobernadores) la estrategia para la internacionalización de la educación superior.

- Realizar estudios de factibilidad y estimaciones de los recursos económicos, materiales y humanos requeridos para el cumplimiento del Programa Nacional de Internacionalización de la Educación Superior.

- Realizar talleres en las IES para promover la incorporación de las estrategias pertinentes de acuerdo a su contexto.

- Establecer indicadores para medir el impacto y el grado de cumplimiento del Programa Nacional de Internacionalización de la Educación Superior.

- Promover la acreditación internacional de programas educativos.

- Impulsar estrategias de formación dirigidas a la profesionalización del personal operativo de las oficinas de relaciones internacionales de las IES.

Esto puede considerarse un intento significativo por proporcionar un marco nacional en el cual sustentar propuestas institucionales; no obstante, persiste la ausencia de una política pública que explicite los mecanismos mediante los cuales se podrían coordinar esfuerzos con la ANUIES y con las mismas IES para impulsar de manera continua y decidida un proceso de internacionalización de la educación superior en México.

Esta es una situación apremiante que debe atenderse, ya que un estudio reciente sobre el estado de la internacionalización en la educación superior en México mostró que las políticas públicas en educación superior en nuestro país no consideran a la internacionalización como rasgo distintivo y que "los factores institucionales que inciden favorablemente son variados y algunos dependerán de condiciones intrínsecas y únicas de cada institución” (Camacho, García, Arroyo y Marsán, 2017, pp. 147-148).

La superación de esta situación requiere de la intervención de una política federal que constituya un marco de referencia para las IES y que defina como prioridad a la 
internacionalización, reconociéndola desde la asignación presupuestal y otorgándole significado como elemento de transformación del sistema de educación superior para hacer frente al contexto global. Esto sería una plataforma muy importante y detonante para que las universidades del país se decidan a impulsar un proceso de internacionalización y la incluyan en su agenda de prioridades institucionales.

En el caso particular de las IES michoacanas, Cruz (2015) apuntó que existen factores que limitan la gestión de mecanismos propios para la internacionalización, tales como una limitada capacidad financiera, procesos institucionales poco alineados con las dinámicas globales y una gestión corporativista, de resitencia al cambio y basada en inercias y lógicas político-sindicales. En este contexto, la autora también señaló que

La estrategia de internacionalización está poco organizada y las instituciones no dimensionan con exactitud su importancia y beneficios. La elaboración de estrategias y programas requiere llevar a cabo transformaciones en las estructuras administrativas, mejoras organizacionales y sobre todo requiere de un compromiso mayor que incorpore evaluaciones continuas y la reestructuración interna de las IES [p. 18].

De este análisis puede concluirse que en el contexto nacional existe una conciencia de la importancia de la internacionalización y se asume que debe dejar de tratarse como una actividad marginal e integrarse a las funciones sustantivas universitarias de forma transversal. Con esto las IES podrían estar mejor preparadas para enfrentar eficaz, eficiente y competitivamente los desafíos que caracterizan al ámbito de la educación superior en la actualidad. Así mismo es muy importante que las universidades asuman un liderazgo en la gestión de una política nacional y presupuestal para la internacionalización ante los gobiernos locales y federal, pues aún hay graves carencias en este aspecto.

\section{El contexto interno.}

Para analizar las condiciones de la internacionalización al interior de la Universidad Michoacana se tomó como guía el cuestionario que se aplicó en la I Encuesta Regional sobre Tendencias de la Internacionalización en Educación Terciaria en América Latina y el Caribe, en virtud de ser un instrumento que incluye todas las estrategias que conforman un proceso de internacionalización comprehensivo o integral (GacelÁvila y Rodríguez-Rodríguez, 2018b).

Dicho cuestionario, y por consiguiente el análisis, se divide en once temas: información y perfil institucional; misión y plan institucional de desarrollo; plan estratégico de internacionalización; oficina de internacionalización; política de enseñanza de idiomas; convenios de colaboración académica; internacionalización del currículo; internacionalización de la investigación; movilidad de estudiantes; evaluación de indicadores de desempeño y beneficios, y riesgos de la internacionalización. 


\section{Información y perfil institucional.}

La Universidad Michoacana es una de las 35 universidades públicas estatales mexicanas (DGESU, 2019). Fue establecida en 1917 pero sus antecedentes históricos se remontan a la época Colonial. Actualmente atiende una matrícula total de 49,647 alumnos en tres niveles educativos (bachillerato, licenciatura y posgrado); cuenta con 1,287 profesores de tiempo completo, de los cuales 26\% pertenece al Sistema Nacional de Investigadores (DGESU-SEP, 2019), y es una institución orientada a la docencia y a la investigación.

La oferta académica de nivel superior consta de 37 programas de licenciatura, cuatro de especialidad, 31 de maestría y 14 de doctorado, en las siguientes áreas del conocimiento: ciencias de la salud, ciencias económico-administrativas, derecho, ciencias exactas, metalurgia y materiales, humanidades, ingenierías y arquitectura, ciencias biológico-agropecuarias y ciencias agropecuarias (UMSNH, 2014).

La cobertura de matrícula de nivel superior es, principalmente, estatal (cuenta con extensiones en nueve municipios del estado de Michoacán, además del campus ubicado en la ciudad de Morelia); no obstante, cada ciclo escolar se registra ingreso de estudiantes originarios de otras entidades del país, como Guanajuato, Guerrero, Chiapas, Estado de México, Oaxaca, Veracruz, Jalisco e Hidalgo (UMSNH, 2014).

\section{Misión y plan institucional de desarrollo.}

El enunciado que describe la misión de la Universidad Michoacana no hace referencia a la internacionalización, y en el plan de desarrollo institucional se menciona de manera tangencial por su incidencia en el mundo laboral y como un rasgo característico del contexto actual entre los elementos del modelo educativo Nicolaita: "la vida moderna caracterizada por la interculturalidad, la rapidez en el flujo de la información, la conformación de redes de colaboración, así como el ensanchamiento de la vinculación y la internacionalización de los grupos de trabajo" (UMSNH, 2010, p. 30). Sin embargo, entre las políticas, programas y ejes estratégicos que componen el modelo educativo no se encuentran objetivos, metas ni acciones específicas en materia de internacionalización y tampoco es un tema que se encuentre presente, de manera explícita, en el discurso oficial de las autoridades universitarias.

\section{Plan estratégico de internacionalización.}

No ha sido elaborado un plan de internacionalización con estrategias, objetivos y metas, a nivel institucional ni por facultades, lo que dificulta la sostenibilidad, en el largo plazo, de cualquier iniciativa que pudiera ser promovida o emprendida como estrategia de internacionalización; particularmente, una de las consecuencias de no ser considerada entre las prioridades definidas a nivel institucional es la falta de financiamiento (Stallivieri y Tabajara, 2020). Algunos autores han señalado que la existencia 
de una política y/o plan estratégico de internacionalización es un pre-requisito para el desarrollo de estrategias de internacionalización (Hernández y Amador, 2015, citados en Galaviz, 2020). En ese sentido, el cambio organizacional es una de las variables fundamentales para el desarrollo y diversificación de estrategias para la internacionalización y para el fortalecimiento de la calidad de los procesos sustantivos universitarios.

\section{Oficina de internacionalización.}

La Universidad Michoacana no cuenta con una oficina de internacionalización en su estructura organizacional. Actualmente los asuntos relacionados con la movilidad académica y los convenios internacionales son atendidos por personal que labora dentro de la DVD, instancia dependiente de la Secretaría de Difusión Cultural y Extensión Universitaria (tercer nivel en el organigrama institucional actual). Tampoco existen responsables de internacionalización por Facultad; no obstante, existe un responsable de vinculación que, entre otras cosas, se encarga de atender a los estudiantes de sus programas académicos que tienen interés por llevar a cabo una movilidad.

Dentro de la DVD existen dos áreas relacionadas con la internacionalización: convenios internacionales y movilidad, las cuales están a cargo de personal administrativo que cuenta con más de diez años de experiencia en el cargo, aunque no tiene estudios de posgrado ni preparación formal en educación superior internacional.

Estas áreas no cuentan con asignación presupuestal ni recursos propios, sus gastos son solventados con recursos de la DVD (según información recopilada mediante entrevistas a informantes clave). Incluso la difusión de información se realiza a través del sitio web de esta dependencia universitaria y los datos que se ofrecen solo se limitan a dar a conocer algunas cifras sobre movilidad entrante y saliente y los países con los que se tiene algún tipo de convenio; esta información solo se proporciona en idioma español.

No existen suscripciones a asociaciones internacionales y/o de cooperación académica a nivel institucional ni de directivos. Tampoco se participa en ferias y eventos de educación internacional en el extranjero.

\section{Política de enseñanza de idiomas.}

En el plan de desarrollo institucional (PDI) de la Universidad Michoacana se menciona que la enseñanza de idiomas es fundamental para la formación integral de los estudiantes, ya que "proporciona herramientas que van más allá del conocimiento de otra lengua, buscando la sensibilización a través del contacto con otras culturas, el acceso a la información científica y cultural de vanguardia" (UMSNH, 2010, p. 20). Sin embargo, no existe una política específica para la enseñanza de idiomas y el dominio del inglés es un requisito de ingreso y/o egreso solo para los programas de posgrado (según información vertida en las entrevistas). 
En algunos programas de licenciatura se ofrecen cursos de inglés técnico como asignatura obligatoria dentro de la malla curricular (los programas de licenciatura en Administración y Comercio Exterior, por ejemplo). Adicionalmente, el Departamento de Idiomas de la universidad ofrece cursos de alemán, árabe, chino, coreano, francés, inglés, italiano, japonés, latín, portugués, p’urhépecha y ruso (UMSNH, 2020), aunque, al ser cursos extracurriculares, solo algunos estudiantes se interesan y benefician de esta oferta.

\section{Convenios de colaboración académica.}

La Universidad Michoacana ha signado diversos convenios con otras IES del mundo, “orientados principalmente al desarrollo conjunto de proyectos en líneas específicas de generación y aplicación del conocimiento, a la movilidad de estudiantes y profesores y a la conformación de redes de diversos tipos” (UMSNH, 2010, p. 45).

Actualmente se cuenta con 186 convenios vigentes de este tipo. Las regiones del mundo en donde se concentra la mayor cantidad son Europa Occidental (44\%) y América Latina y el Caribe (42\%). En la primera destaca que tres de cada cuatro son convenios firmados con IES españolas y, en la región latinoamericana y caribeña, destacan las universidades colombianas con un $24 \%$, chilenas $20 \%$, cubanas $18 \%$ y peruanas 16\%. Los convenios con América del Norte solo representan el 10\%, y en el caso de Asia el 5\% del total, mientras África, Medio Oriente y Oceanía son regiones con las que no se han establecido lazos de colaboración hasta el momento (información que consta en bases de datos institucionales, proporcionada por los informantes clave).

\section{Internacionalización del currículo.}

Aunque la Universidad Michoacana no cuenta con una política institucional para la internacionalización del currículo, en el PDI se menciona que "atentos a la formación integral de los estudiantes universitarios", se ha proyectado "la vinculación y la movilidad académica, para favorecer el intercambio de conocimientos, experiencias de investigación y la cooperación internacional" (UMSNH, 2010, p. 19), y define dos acciones: "generalizar el sistema de créditos e incluir el concepto de movilidad en la legislación institucional" (UMSNH, 2010, p. 65) y "suscribir convenios interinstitucionales de movilidad estudiantil" (UMSNH, 2010, p. 66), orientadas a cumplir el objetivo de "contar con una formación en los diversos niveles educativos actualizada, flexible y de calidad; innovadora del conocimiento, acorde a la demanda social, a su distribución geográfica y al desarrollo regional y nacional" (UMSNH, 2010, p. 65).

Por otra parte, la limitación de recursos presupuestales para apoyar a la movilidad estudiantil, así como la falta de formalización institucional de esta, son reconocidas como debilidades institucionales en el mismo PDI. Sin embargo, podría agregarse 
una más: la consideración de la movilidad internacional como única estrategia para cumplir con el objetivo mencionado, ya que otras estrategias que podrían contribuir al fortalecimiento e internacionalización del currículo se están dejando al margen, como pueden ser: movilidad entrante de estudiantes, invitación de profesores extranjeros, impartición de asignaturas en las que se estudien otras culturas, inclusión de programas/cursos curriculares impartidos en un idioma distinto al español, codirección de tesis con investigadores extranjeros, cursos impartidos en colaboración con instituciones extranjeras, programas de grado conjunto y/o doble con instituciones extranjeras, por citar algunos ejemplos (Gacel-Ávila y Rodríguez-Rodríguez, 2018b).

\section{Internacionalización de la investigación.}

A pesar de que en el PDI se enuncia la creación de "un programa para la ampliación de convenios nacionales e internacionales que incluya la movilidad de profesores para el fomento de la docencia y la investigación” (UMSNH, 2010, pp. 71-72), con el objetivo de fortalecer el perfil docente y la calidad académica y de la investigación, esta sigue siendo una tarea pendiente.

La información obtenida a través de las entrevistas a informantes clave indica que no existe un programa institucional de financiamiento para la movilidad de académicos y tampoco para proyectos internacionales de investigación. Incluso, la dependencia universitaria encargada de la movilidad no cuenta con información precisa en torno a la cantidad de investigadores que llevan a cabo estancias en el extranjero, las publicaciones que estos realizan fuera de México, o de las redes internacionales de investigación en las que participan. En tales circunstancias, es complicado dar seguimiento a las actividades de internacionalización que pudieran estarse realizando en el ámbito de la investigación

\section{Movilidad de estudiantes.}

Aunque la Universidad Michoacana no cuenta con un programa estratégico para la movilidad estudiantil, esta es una actividad que se ha realizado de manera continua por lo menos durante los últimos diez años, según registros del área administrativa responsable de la operatividad de la movilidad. La información obtenida de las entrevistas a informantes clave permitió identificar que las principales modalidades de movilidad internacional de estudiantes que se promueven son: cursar asignaturas, estancias de investigación y estudios de posgrado.

Asimismo, las personas entrevistadas señalaron que los principales países destino de los estudiantes que realizan movilidad son hispanoparlantes, entre los que destacan Colombia, Chile, Perú, Argentina y España. En contraparte, los estudiantes extranjeros que recibe la Universidad Michoacana son, principalmente, de origen colombiano y chileno. 
Entre las principales dificultades para impulsar la movilidad estudiantil hacia otras regiones del mundo, señaladas por las entrevistadas, se encuentran la falta de dominio de un segundo idioma y la escasez de recursos financieros, tanto de los propios estudiantes como institucionales, ya que no se cuenta con un programa de becas de apoyo a la movilidad. En cuanto a la recepción de estudiantes extranjeros, los principales factores que la limitan son la escasa promoción de la oferta académica de la Universidad Michoacana en ferias y/o eventos en el extranjero, así como la falta de participación de la institución en redes y organismos que promueven la movilidad estudiantil y la internacionalización de la educación superior.

El área encargada de la movilidad cuenta con un programa de difusión de opciones, requisitos y procedimientos para llevar a cabo una movilidad y, aunque se ofrece preparación previa a los estudiantes que han sido seleccionados para realizar una estancia en el extranjero, esta no resulta suficiente debido a que no incluye la preparación en lenguas extranjeras.

\section{Evaluación de indicadores de desempeño.}

La información obtenida mediante entrevistas permitió conocer que no se cuenta con un sistema de evaluación de indicadores de desempeño del proceso de internacionalización y tampoco existe un programa de evaluación de los resultados o impactos de la movilidad académica, a pesar de que en el PDI se menciona que

La evaluación y seguimiento [...] incluye a la totalidad de las áreas administrativas y académicas [...] Hacer evaluación y seguimiento en nuestra institución implica, por una parte, contar indefectiblemente con un Plan de Desarrollo Institucional, que dé rumbo y movimiento a esta y otras necesidades; pero también implica construir un Sistema Institucional de Indicadores, tarea confiada a la Comisión de Planeación Universitaria, por medio del cual se generará información acerca del desempeño de las áreas administrativas y académicas que permitirá que estas, por medio de su evaluación, conozcan el cumplimiento de sus avances y metas planteadas en sus Programas Operativos Anuales y/o en sus Planes de Desarrollo particulares. La información generada por este sistema servirá asimismo para reportar a la Administración Central sobre el rumbo de la universidad en el contexto de lo planeado. El Sistema Institucional de Indicadores será de igual forma un importante recurso para elaborar informes para otras instancias e instituciones acerca del progreso de la universidad, para la realización de los informes de la Administración Central y para la formulación de proyectos y programas generales y específicos [UMSNH, 2010, p. 84].

\section{Beneficios y riesgos de la internacionalización.}

No se cuenta con información oficial disponible que dé cuenta de un análisis en torno a los riesgos/beneficios que atañen a la internacionalización en la Universidad Michoacana, ni de una valoración de los impulsores externos que ejercen presiones para impulsarla. Según un estudio realizado en el 2015 acerca de los procesos organizacionales para la internacionalización en las principales IES del estado de 
Michoacán, el anterior rectorado consideraba que la internacionalización, en la Universidad Michoacana, era

...una actividad importante pero no sustancial: no forma parte de su misión y objetivos. El propósito central está enfocado a cumplir con una función social de lugar incluyente y democrático que logre consolidar la calidad académica de los posgrados. Las actividades de internacionalización acompañan este crecimiento pero, prácticamente, funcionan con apoyos externos... [Cruz, 2015, p. 10].

Actualmente no se conoce cuál es la postura desde la administración central, ya que no se cuenta con informes oficiales recientes y sigue operando el PDI 2010-2020.

Como puede observarse, este análisis del contexto interno demuestra que en la Universidad Michoacana hay indicios de algunas acciones de internacionalización, sin embargo se trata de esfuerzos desarticulados, que no han sido planificados en torno a un proceso institucional y centrados en la movilidad estudiantil saliente. Para rebasar esta situación es necesario reconocer formalmente la relevancia de la dimensión internacional y trabajar, de forma decidida, en un plan estratégico con asignaciones presupuestales acordes que orienten el proceso que implica su integración en la razón de ser de esta universidad, así como en sus funciones sustantivas y en su estructura organizacional.

Esto permitiría atender una de las necesidades más apremiantes al interior de la Universidad Michoacana que, de acuerdo a la información recopilada mediante entrevistas, consiste en revertir el agudo deterioro institucional que ha tenido severas y diversas manifestaciones: caída de la matrícula, desarticulación entre los programas y niveles educativos que se ofrecen, desvinculación al interior de la misma universidad y con la sociedad, por citar algunos ejemplos.

Esta situación es, sin duda alguna, crítica e insostenible, incluso a corto plazo. Para superarla es menester atender sus causas, reconfigurando las formas en que se ha venido ejerciendo la gobernanza institucional y dejando atrás prácticas como el pragmatismo, la tecnocracia y la verticalidad en la toma decisiones.

Puede hacerse, de este contexto adverso, una ventana de oportunidad que propicie un cambio institucional y un ambiente propicio para la generación de un debate acerca de la necesidad y objetivos de la internacionalización en esta Universidad.

\section{Conciencia.}

El impulso del proceso de internacionalización, como se ha mencionado, requiere del involucramiento de todos los ámbitos, sectores y niveles institucionales. Si bien es un esfuerzo que debe liderar el rector, con la participación de un equipo conformado por especialistas y directivos de áreas clave para asegurar una adecuada implementación de la estrategia, queda claro que, para tener impacto sistémico, la internacionalización debe ser asumida como un compromiso colectivo de toda la comunidad universitaria. 
Por tanto, una de las primeras tareas para impulsar de manera institucional el proceso de internacionalización consiste en desarrollar una estrategia de sensibilización por etapas: primero entre funcionarios de primer nivel, enseguida orientarla al personal de las áreas académico-administrativas vinculadas al proceso, y posteriormente convocar a la planta docente.

En el caso de la Universidad Michoacana este recorrido organizacional implicaría a la Rectoría, la Secretaría General, la Secretaría Académica, la Secretaría Administrativa y al Consejo Universitario (como primer nivel de decisión), continuaría con la DVD, la Coordinación del Departamento de Idiomas, la Coordinación General de Educación Continua, a Distancia y Abierta, la Dirección de Control Escolar, la Coordinación para la Evaluación y Acreditación, la Coordinación de Responsabilidad Social y Formación Docente, la Coordinación General de Estudios de Posgrado, la Coordinación General de Educación Superior y la Coordinación General de la División del Bachillerato (en un segundo nivel de decisión), y finalmente habría que llegar al nivel de facultades e institutos, sensibilizando a sus docentes, personal directivo y Consejos Técnicos, dado que estos constituyen los espacios de análisis, discusión y toma de decisiones en torno a la planeación académica y los contenidos curriculares de cada programa que se oferta en esta universidad.

En esta primera etapa, la concientización acerca de la importancia y beneficios de impulsar un proceso de internacionalización permitiría entablar un diálogo participativo con diversos actores y grupos de interés, condición fundamental para la consolidación de acciones, ya que, en la medida en que son tomadas en cuenta las opiniones y propuestas de los miembros de la comunidad universitaria, es posible su involucramiento a partir de su propia convicción.

En este sentido, se estarían sentando las bases para una siguiente fase en el proceso de internacionalización, que implicaría llevarla a las aulas, al proceso de enseñanzaaprendizaje, lo que constituye otro reto importante e incluye a otros implicados: los estudiantes.

\section{Compromiso.}

Una vez que se haya logrado permear la importancia de la internacionalización entre la comunidad universitaria, es preciso que las autoridades asuman un compromiso institucional que articule la discusión y sensibilización previas con el diseño e implementación de la política de internacionalización. Idealmente, ese compromiso deberá quedar explícito a través del diseño, rediseño o adecuación del marco institucional vigente, a fin de contar con las estructuras normativa y funcional adecuadas para impulsar el proceso de internacionalización.

Para el caso de la Universidad Michoacana, esto quiere decir que se deberá contar con la aprobación del documento escrito que contiene la política de internaciona- 
lización, por parte del H. Consejo Universitario; así mismo, la misión, la visión, los valores y el modelo educativo nicolaita deberán contener declaraciones explícitas acerca de la relevancia y el compromiso que se asume con la internacionalización. Además, el plan de desarrollo institucional deberá contener lineamientos, estrategias, objetivos y metas explícitas en torno a la internacionalización, así como especificar los recursos que se destinarán para cumplirlas; todo ello en concordancia con la política de internacionalización.

\section{Planeación.}

La elaboración de un plan estratégico de internacionalización es elemental para impulsar el proceso y sostenerlo a largo plazo. Se trata de un documento institucional que debe definir, integrar y relacionar de forma explícita diversos elementos en torno a la internacionalización: visión a largo plazo; misión y valores que orientarán el trabajo para lograr el cumplimiento de lo establecido en la visión y misión; objetivos de corto, mediano y largo plazo, acompañados de las metas que se pretenden alcanzar con cada uno de ellos y los indicadores que permitirán evaluar el nivel de avance obtenido en cada plazo establecido; estrategias y acciones específicas que es necesario llevar a cabo para cumplir con los objetivos y, finalmente, un sistema de evaluación y seguimiento que permita detectar la necesidad de hacer ajustes y reorientar estrategias, de tal modo que se logre el cumplimiento de los objetivos que se ha planteado la institución.

De acuerdo al análisis del contexto que se ha realizado, es necesario que la Universidad Michoacana integre la dimensión internacional en el texto que enuncia su misión y visión institucional, de tal forma que se haga explícito su compromiso de formar ciudadanos globales, respetuosos de la diversidad cultural y capaces de contribuir al desarrollo humano sostenible.

Asimismo es preciso establecer objetivos específicos en torno a la internacionalización en los diferentes ámbitos que implica el cumplimiento de sus funciones sustantivas. Por ejemplo, en materia de gestión, podría plantearse el objetivo de constituir una plataforma administrativa y organizacional que propicie la viabilidad y sostenibilidad del proceso de internacionalización; en el ámbito de formación, el objetivo podría ser propiciar las condiciones académicas para que el alumnado desarrolle competencias que le permitan desempeñarse adecuadamente en el entorno local e internacional; en investigación, el objetivo podría estar orientado a fomentar el fortalecimiento del perfil y proyección internacional de la investigación que se realiza desde la Universidad Michoacana, y en cuanto a extensión y difusión, el objetivo podría encauzarse a contribuir al fortalecimiento de la proyección internacional de la Universidad Michoacana.

Algunas estrategias de partida que podrían desarrollarse para cumplir con el objetivo enunciado en el ámbito de gestión incluirían: capacitación al personal encargado de 
la internacionalización; disposición de financiamiento propio y suficiente (en cantidad y permanencia) para implementar el plan de internacionalización; participación en redes y asociaciones que promueven la internacionalización de la educación superior en México y en otros países; asistencia a eventos internacionales que permitan incrementar y consolidar vínculos con universidades de otros países; creación de un banco de datos sobre internacionalización y de un sistema de indicadores para la evaluación de la política de internacionalización.

En cuanto al ámbito de formación, algunas estrategias que podrían contribuir al cumplimiento de su objetivo serían: integrar el idioma inglés en los cursos curriculares de licenciatura; promover una mayor participación de estudiantes y profesores en convocatorias de movilidad internacional, y diversificar las posibilidades de aprendizaje intercultural para todos los estudiantes, principalmente para aquellos que no tienen la oportunidad de realizar una movilidad.

Para impulsar la internacionalización de la investigación, podría empezarse por financiar proyectos de investigación de alcance internacional; contribuir a la conformación y consolidación de redes de investigación científica internacional, e incentivar una mayor participación de académicos en convocatorias internacionales para la obtención de financiamiento para la investigación científica.

Finalmente, sería necesario estimular la comprensión de realidades culturales diversas a través de procesos de gestión cultural, en el ámbito de la extensión y difusión, lo cual implicaría, por ejemplo: realizar diálogos interculturales en las diferentes dependencias académicas y niveles educativos (bachillerato, licenciatura y posgrado); realizar, en conjunto con otras dependencias, ferias y jornadas de internacionalización; crear una red de apoyo a estudiantes en movilidad; promover los programas educativos y cursos de la Universidad Michoacana mediante redes sociales para la atracción de estudiantes extranjeros.

\section{Operacionalización.}

Como se ha dicho, la planeación del proceso de internacionalización es de suma importancia, pero dar el paso del plan a la acción requiere definir responsables y asignarles atribuciones y tareas, por lo que, en el caso de la Universidad Michoacana, deberá conformarse un equipo de trabajo responsable de operar la política de internacionalización a nivel central, así como un comité de internacionalización, con las siguientes características y facultades:

- Contar con representantes de los diferentes sectores de la comunidad universitaria (estudiantes, profesores, personal administrativo) y de las diferentes áreas del conocimiento; es importante que quienes integren este comité sean sensibles y conscientes de la relevancia de la internacionalización de la educación superior; 
- tener legitimidad y poder de convocatoria para coordinar trabajos con otras áreas, dependencias y niveles de la administración universitaria, así como representatividad institucional para vincularse con actores externos. Esto significa que cada miembro del comité deberá contar con un nombramiento oficial que lo acredite como tal y se debe asegurar que toda la comunidad universitaria sepa de su existencia y funciones;

- disponer de acceso a información institucional que requiera para el ejercicio de sus funciones y/o proyectos;

- contar con un espacio físico, material y equipo de oficina para el desempeño de sus funciones (sería idóneo que esté reconocido, incluso, en el organigrama institucional);

- tener asignado un presupuesto anual específico para cumplir con los objetivos y metas establecidas en la política institucional;

- contar con un manual de procedimientos;

- tener participación en los procesos de planeación para hacer llegar propuestas de mejora, y, finalmente,

- contar con mecanismos para el cumplimiento de transparencia y rendición de cuentas, como lo establece la normatividad en la materia.

\section{Implementación.}

Una vez definido el personal responsable de operar el plan estratégico de internacionalización, es posible avanzar en la implementación. Esta consiste en poner en marcha el plan y en funciones al equipo constituido para ese fin, en coordinación con las áreas académicas y administrativas que se requiera, según el programa y/o acciones específicas a implementar.

En el caso de la Universidad Michoacana, en esta fase se involucrarían las coordinaciones correspondientes a cada nivel educativo, las áreas que llevan a cabo funciones de formación docente y las direcciones de facultades e institutos, ya que es necesario seguir los canales institucionales que permitan llegar hasta el aula, que es el espacio físico en el que se implementan, en última instancia, los programas y estrategias de internacionalización.

No obstante, llegar a este nivel implica otros retos para la universidad, ya que tendría que diseñarse un programa específico para la internacionalización del currículo, derivado de los objetivos de internacionalización a mediano y largo plazos.

A corto plazo, la implementación incluiría la ejecución de acciones estratégicas a nivel de facultades e institutos, en concordancia con el plan de internacionalización implementado desde la administración central. Para llevar a cabo esta tarea será necesario que se designe a una persona como enlace de comunicación con el comité de internacionalización que opere a nivel central. 
Evaluación.

La creación de un sistema de evaluación de indicadores de desempeño del proceso de internacionalización, así como el diseño de un programa de evaluación de los resultados de movilidad de profesores y de estudiantes, constituye una tarea fundamental tanto en términos de la posibilidad de hacer un seguimiento para la mejora continua de procesos y actividades, como en términos de la transparencia y rendición de cuentas. Gacel-Ávila precisó que "la internacionalización implica un proceso de evaluación y seguimiento sistemático dentro del proceso de evaluación global de la institución” y que sus impactos deben ser evaluados en diferentes ámbitos para impulsar su mejora continua (2003, p. 328).

En las últimas dos décadas se han diseñado distintas metodologías orientadas a evaluar los impactos de la internacionalización, pero esos trabajos aún resultan insuficientes para conocer a detalle el estado de la internacionalización de las IES, debido a que las características de los sistemas de educación superior varían de un país a otro.

Quinteiro (2020) puntualizó algunos factores que han dificultado medir los resultados de la internacionalización en la educación superior: falta de consenso sobre los componentes que debería incluir la medición; falta de inclusión de todas las estrategias de internacionalización en los instrumentos de evaluación, pues muchos de ellos se centran solo en la movilidad estudiantil; heterogeneidad de indicadores que dificulta la comparabilidad internacional; inclusión de parámetros que son alcanzables solo para ciertas IES y, por último, la dificultad para recopilar información comparable.

Para atender a la necesidad y exigencia que tienen las IES por medir el impacto que tienen sus esfuerzos de internacionalización, en términos de su contribución a la pertinencia y calidad de la educación, el autor propuso que "el proceso de internacionalización también puede ser asumido por las propias IES bajo un modelo de autorregulación que comprenda la autoevaluación y la evaluación por pares académicos o instituciones equivalentes" (Quinteiro, 2020, p. 12), la cual podría ser una alternativa para la Universidad Michoacana, una vez definida una política de internacionalización a nivel institucional.

\section{Refuerzo.}

Para motivar y reforzar la participación de los docentes y los estudiantes en el proceso de internacionalización es necesario generar incentivos y reconocimientos. Por ejemplo, podría destinarse un fondo, desde la Coordinación de la Investigación Científica, para contribuir financieramente en proyectos de investigación que coordinen profesores de la Universidad Michoacana con pares internacionales; también podría asignarse una puntuación, dentro del Programa de Estímulos al Desempeño del Personal Docente, a la participación de los docentes en actividades directamente vinculadas al cumplimiento de los objetivos de la política de internacionalización. 
Asimismo podría otorgarse un reconocimiento y un estímulo económico a los estudiantes que participen en actividades de internacionalización.

\section{Esfuerzo de integración.}

Este elemento constituye el cierre del proceso de internacionalización y, al mismo tiempo, el inicio de un nuevo ciclo, ya que los elementos que constituyen las fases anteriores deben lograr un nivel de integración que permita garantizar que las estrategias que se han implementado tengan un verdadero impacto en las funciones sustantivas de la universidad. En palabras de Aguilar y Riveros:

...en la actualidad no es suficiente que una universidad participe en una gran cantidad de actividades, sino en las que sean requeridas para fortalecer su papel como formadora de grupos profesionales idóneos para una sociedad que los requiere de tal manera que se asegure de la calidad de la educación que imparten y de los servicios que ofrecen, en función de su misión institucional [2017, pp. 12-13].

\section{Conclusiones}

La Universidad Michoacana no ha integrado la dimensión internacional en sus funciones sustantivas, cuando mucho, ha implementado algunos programas de internacionalización de manera marginal, aislada y sin un objetivo claro, como es el caso de la movilidad de profesores y alumnos.

Esto sucede por dos razones, la primera es la ausencia de un liderazgo consciente de la importancia que el tema tiene, no solo como una de las grandes tendencias de la educación superior sino como imperativo para el cumplimiento de las funciones sustantivas universitarias. La segunda razón, como consecuencia de la primera, es la inexistencia de una política institucional explícita que oriente, coordine y formalice los esfuerzos por impulsar un proceso de internacionalización.

Como se ha señalado, tanto el debate nacional como internacional que existe actualmente en torno a las características, objetivos y desafíos de la educación superior coincide en destacar su naturaleza como medio para lograr la emancipación social, garantizar el respeto a los derechos humanos y reducir las brechas que existen al interior de los países y entre ellos, y en este contexto se hace especial énfasis en el destacado papel de la internacionalización para contribuir al alcance de dichos objetivos.

En apego a estos principios, los elementos de partida para impulsar el proceso de internacionalización en la Universidad Michoacana que aquí se han descrito tienen como fundamento la convicción de que es necesario fortalecer en la formación de sus estudiantes tanto los aprendizajes técnicos que requieren para el ejercicio de su profesión como los aprendizajes que les permitan desarrollar una postura crítica y con un sentido ampliamente social. Esto último es menester en un contexto que nos resulta adverso por las condiciones que ha impuesto la implementación de políticas 
de corte neoliberal, que han sometido a las instituciones de educación superior a una dinámica que supedita lo educativo a lo económico. La Universidad Michoacana debe ocuparse no solo de mejorar la calidad y cobertura de la educación superior sino, además, de transformar prácticas que reproducen desigualad y discriminación, tal como lo establece su ley orgánica y su misión institucional.

El momento actual ofrece una importante ventana de oportunidad para modificar el estado de las cosas aquí descrito, pues se está preparando el nuevo Plan de Desarrollo Institucional que entraría en vigencia en 2021, en el cual debería ser integrada la dimensión internacional.

Por último, es importante señalar que la reconfiguración académica, organizativa, administrativa y política es fundamental para sentar las bases e impulsar el proceso de internacionalización, y es quizá, al mismo tiempo, el principal reto al que tenga que enfrentarse esta institución.

\section{REFERENCIAS}

Aguilar, Y., y Riveros, A. (2017). La internacionalización de la educación superior: concepto y evolución del modelo en la Universidad de Costa Rica. Revista Educación, 41(1), 1-31. Recuperado de: https:/ /www. researchgate.net/publication/312398502_La_internacionalizacion_de_la_educacion_superior_ Concepto_y_evolucion_del_modelo_en_la_Universidad_de_Costa_Rica.

ANUIES [Asociación Nacional de Universidades e Instituciones de Educación Superior] (2018). Visión y acción 2030. Propuesta de la ANUIES para renovar la educación superior en México. Recuperado de: https:// www.google.com $/$ url? sa $=t \& r c t=j \& q=\& e s r c=s \& s o$ urce $=$ web\&cd $=1 \& \mathrm{cad}=$ rja\&uact $=8 \&$ ved $=2 \mathrm{ahUK}$ Ewjqk73i8q_mAhVLKKwKHSIAAQAQFjAAegQ IBxAC\&url=http $\% 3 \mathrm{~A} \% 2 \mathrm{~F} \% 2 \mathrm{Fwww}$.anuies.mx $\%$ 2Fmedia $\% 2$ Fdocs $\% 2$ Favisos $\% 2$ Fpdf $\% 2 F V I S I O N$ _Y_ACCION_2030.pdf\&usg=AOvVaw1cJtrRuapb WRGYVnxwUx2x.

Camacho, M., García, D., Arroyo, C., y Marsán, E. (2017). El estado de la internacionalización en la educación superior en México. México: British Council México/Centro de Investigación y Docencia Económicas. Recuperado de: https://issuu.com/britishcouncilmexico/ docs/03_iesm_ok_lap_final_compressed $11 \mathrm{mb}$.

CRES [Conferencia Regional de Educación Superior] (2018). Declaración. III Conferencia Regional de Educa- ción Superior para América Latina y el Caribe. Instituto Internacional de la UNESCO para la Educación Superior en América Latina y el Caribe/Universidad Nacional de Córdoba. Recuperado de: https:/ /www. iesalc.unesco.org/2019/02/20/declaracion-final-dela-iii-conferencia-regional-de-educacion-superior-enamerica-latina-y-el-caribe-cres-2018/.

Cruz, E. (2015). Procesos organizacionales para la internacionalización de la educación: el caso de las principales instituciones de educación superior en Michoacán. Red sobre Internacionalización y Movilidades Académicas y Científicas. Recuperado de: https://www.rimac.mx/procesosorganizacionales-para-la-internacionalizacion-de-laeducacion-el-caso-de-las-principales-institucionesde-educacion-superior-en-michoacan/.

De Wit, H., Hunter, F., Howard, L., y Egron-Polak, E. (2015). Internationalisation of Higher Education. Bruselas: European Parliament, Committee on Culture and Education. Recuperado de: https://www.europarl. europa.eu/RegData/etudes/STUD/2015/540370/ IPOL_STU(2015)540370_EN.pdf.

DGESU [Dirección General de Educación Superior Universitaria] (2019, ago. 10). Universidades públicas estatales. Recuperado de: https://www.dgesu.ses.sep. gob.mx/UPES.html.

DGESU-SEP [Dirección General de Educación Superior Universitaria-Secretaría de Educación Pública] (2019, 
nov. 1). UMSNH Universidad Michoacana de San Nicolás de Hidalgo. Plataforma en transparencia. Rendición de cuentas. Recuperado de: https://sep.subsidioentransparencia.mx/2019/subsidio-ordinario/universidad/UMSNH.

Egron-Polak, E. y Hudson, H. (2014). Internationalization of higher education: Growing expectations, fundamental values. IAU $4^{\text {th }}$ Global Survey. International Association of Universities.

Gacel-Ávila, J. (2003). La internacionalización de la educación superior. Paradigma para la ciudadanía global. Universidad de Guadalajara.

Gacel-Ávila, J., y Rodríguez-Rodríguez, S. (2018a). La internacionalización de la educación terciaria en América Latina y el Caribe: avances, tendencias y visión a futuro. En J. Gacel-Ávila (coord.), Educación superior, internacionalización e integración en América Latina y el Caribe. Balance regional y prospectiva (pp. 57-88). Córdoba, Argentina: Instituto Internacional de la UNESCO para la Educación Superior en América Latina y el Caribe/Universidad Nacional de Córdoba. Recuperado de: https://www.iesalc. unesco.org/2019/07/17/coleccion-cres-2018-educacion-superior-internacionalizacion-e-integracionen-america-latina-y-el-caribe-balance-regional-yprospectiva/.

Gacel-Ávila, J., y Rodríguez-Rodríguez, S. (2018b). Internacionalización de la educación superior en América Latina y el Caribe. Un balance. Universidad de Guadalajara/ Instituto Internacional para la Educación Superior en América Latina y el Caribe (IESALC-UNESCO)/ Benemérita Universidad Autónoma de Puebla. Recuperado de: http://obiret-iesalc.udg.mx/es/ publicaciones-propias/internacionalizacion-de-laeducacion-superior-en-america-latina-y-el-caribe-un.

Galaviz, B. (2020). Internacionalización responsable: más allá de una evaluación formal y técnica de la internacionalización de la educación superior. En F. Fernández y A. Velázquez (coords.), La internacionalización de la universidad pública: retos y tendencias. Una visión desde la UNAM (pp. 71-82). Universidad Nacional Autónoma de México. Recuperado de: https://www. researchgate.net/publication/345000862_Librola_internacionalizacion_de_la_universidad_publica_ retos_y_tendencias_una_vision_desde_la_UNAM.
Knight, J. (2012). Concepts, rationales, and interpretive frameworks in the internationalization of higher education. En H. d. Darla K. Deardorff, The $S A G E$ handbook of international higher education (pp. 27-42). SAGE Publications. doi: http://dx.doi. org/10.4135/9781452218397.n2.

Madera, L. (2018). Educación y desarrollo sostenible al 2030: internacionalización de la formación docente en América Latina y el Caribe. En J. Gacel-Ávila (coord.), La educación superior, internacionalización e integración regional de América Latina y el Caribe (pp. 89- 110). Instituto Internacional de la UNESCO para la Educación Superior en América Latina y el Caribe [UNESCO-IESALC]/ Universidad Nacional de Córdoba. Recuperado de: http:/ / erasmusplusriesal.org/sites/default/files / libro_la_es_inter_e_integracion_cres.pdf.

Navarrete, Z. (2021). Políticas para la educación secundaria en perspectiva comparada: Costa Rica y Venezuela. En M. A. Navarro-Leal, Z. Navarrete-Cazales y J. R. Rivera Peña (coords.), Políticas educativas. Una mirada internacional y comparada (pp. 97-111). Escuela Normal "Miguel F. Martínez" Centenaria y Benemérita/Sociedad Mexicana de Educación Comparada. Recuperado de: https://www.somec.mx/libro-politicas-educativasuna-mirada-internacional-y-comparada/.

Ordorika, I., y Soley, T. (2018). Ejes para la transformación estratégica de las universidades públicas en América Latina: A 100 años de la Reforma de Córdoba. En P. Hernández Guajardo (coord.), El papel estratégico de la educación superior en el desarrollo sostenible de América Latina y el Caribe (pp. 53-66). Instituto Internacional de la UNESCO para la Educación Superior en América Latina y el Caribe [UNESCO-IESALC]/Universidad Nacional de Córdoba. Recuperado de: https:/ /www.iesalc.unesco.org/2019/07/17/coleccion-cres-2018-elpapel-estrategico-de-la-educacion-superior-en-eldesarrollo-sostenible-de-america-latina-y-el-caribe/.

Quinteiro, J. (2020, mar. 7). La internacionalización de la educación superior: ¿y la medición para cuándo? Recuperado de: https://www.iesalc.unesco.org/2020/03/07/ la-internacionalizacion-de-la-educacion-superior-y-lamedicion-para-cuando/.

Stallivieri, L., y Tabajara, C. (2020). Internacionalización responsable: más allá de una evaluación formal y técnica de la internacionalización de la educación superior. 
Educación Global, 24, 32-46. Recuperado de: http:// obiret-iesalc.udg.mx/es/revista/revista-educacionglobal.

Tünnermann, C. (2018). La internacionalización de la educación superior. Significado, relevancia y evolución histórica. En J. Gacel-Ávila, La educación superior, internacionalización e integración regional de América Latina y el Caribe (pp. 17-39). Córdoba, Argentina: Instituto Internacional de la UNESCO para la Educación Superior en América Latina y el Caribe [UNESCOIESALC]/Universidad Nacional de Córdoba. Recuperado de: https://www.google.com/url?sa $=$ t\&rct $=$ $j \& q=\& e s r c=s \&$ source $=$ web\&cd $=\& v e d=2$ ahUKEwi RzMSNjNfuAhVRmK0KHZ0_A1cQFjAAegQIAR
AC\&url $=$ http $\% 3 \mathrm{~A} \% 2 \mathrm{~F} \% 2$ Ferasmusplusriesal.org $\% 2$

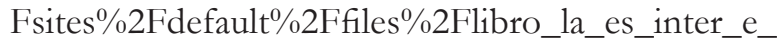
integracion_cres.pdf\&usg $=\mathrm{AOvVaw} 3 \mathrm{dxgO} 4 \mathrm{vLasx}$ QUEtJM9NuQO.

UMSNH [Universidad Michoacana de San Nicolás de Hidalgo] (2010). Plan de Desarrollo Institucional 20102020. Recuperado de: https://www.umich.mx/ normatividad.html.

UMSNH (2014, feb. 3). Universidad Michoacana de San Nicolás de Hidalgo. Recuperado de: http:/ / www.umich. $\mathrm{mx} /$.

UMSNH (2020, feb. 28). Coordinación del Departamento de Idiomas. Recuperado de: http://www.idiomas.umich. $\mathrm{mx} /$ nosotros.html\#antecedentes.

Cómo citar este artículo:

Vega Cano, R. (2021). Primeros pasos hacia la internacionalización en la Universidad Michoacana de San Nicolás de Hidalgo. IE Revista de Investigación Educativa de la REDIECH, 12, e1117. doi: 10.33010/ie_rie_rediech.v12i0.1117. 